\title{
OPEN Dog ownership and adults' objectively-assessed sedentary behaviour and physical activity
}

\author{
Mohammad Javad Koohsari ${ }^{1,2,3 凶}$, Ai Shibata ${ }^{4}$, Kaori Ishii ${ }^{1}$, Sayaka Kurosawa ${ }^{5}$, \\ Akitomo Yasunaga ${ }^{6}$, Tomoya Hanibuchi ${ }^{7}$, Tomoki Nakaya ${ }^{7}$, Gavin R. McCormack ${ }^{1,8,9,10}$ \& \\ Koichiro Oka ${ }^{1}$
}

Evidence suggests a positive effect of dog ownership on physical activity. However, most previous studies used self-reported physical activity measures. Additionally, it is unknown whether owning a dog is associated with adults' sedentary behaviour, an emerging health risk factor. In this study, physical activity and sedentary behaviour were objectively collected between 2013 and 2015 from 693 residents (aged 40-64 years) living in Japan using accelerometer devices. Multivariable linear regression models were used, adjusted for several covariates. The means of total sedentary time and the number of long $(\geq 30 \mathrm{~min})$ sedentary bouts were $26.29 \mathrm{~min} / \mathrm{day}(95 \% \mathrm{Cl}-47.85,-4.72)$ and 0.41 times/day $(95 \% \mathrm{Cl}-0.72,-0.10)$ lower for those who owned a dog compared to those not owning a dog, respectively. Compared with non-owners, dog-owners had significantly higher means of the number of sedentary breaks $(95 \% \mathrm{Cl} 0.14,1.22)$, and light-intensity physical activity $(95 \% \mathrm{Cl} 1.31$, 37.51). No significant differences in duration of long ( $\geq 30 \mathrm{~min}$ ) sedentary bouts, moderate, vigorous, and moderate-to-vigorous-intensity physical activity were observed between dog-owners and nonowners. A novel finding of this study is that owning a dog was associated with several types of adults' sedentary behaviours but not medium-to-high-intensity physical activities. These findings provide new insights for dog-based behavioural health interventions on the benefits of dog ownership for reducing sedentary behaviour.

Pet ownership, particularly dog ownership, is associated with physical and mental health benefits ${ }^{1,2}$. For example, a nationwide cohort study conducted in Sweden found that dog ownership was associated with a lower risk of cardiovascular disease and mortality ${ }^{3}$. Another national study conducted in the USA found that pet ownership (either cat or dog ownership) was associated with lower odds of systemic hypertension ${ }^{4}$. Dog walking is one of the possible mechanisms through which dog ownership may affect people's physical activity and health ${ }^{5,6}$. The health benefits of physical activity have been well-documented ${ }^{7}$, yet most adults are insufficiently active ${ }^{8}$. In the USA, only $22.9 \%$ of adults met national guidelines for aerobic and muscle-strengthening activities ${ }^{9}$. In Japan, population-level physical activity has continuously decreased during the last few decades ${ }^{10}$, and about $38 \%$ of Japanese were insufficiently active in 2016, according to the World Health Organisation report ${ }^{11}$. Alongside individual-based factors, socio-ecological models emphasise the role of social and urban design factors in supporting physical activity ${ }^{12}$. Given the prevalence of dog ownership in households in Japan (17\% $)^{13}$ and other countries such as Canada $(35 \%)^{14}$, USA $(48 \%)^{15}$ and Australia $(38 \%)^{16}$, physical activity promotion strategies that target dog-owners could have a significant impact on population health.

There is growing evidence over the positive effects of dog ownership on people's physical activity ${ }^{17-19}$. For instance, a study conducted in the UK found that dog-owners were more likely to undertake more recreational walking and have higher odds of meeting physical activity guidelines, compared with non-owners ${ }^{20}$. Another

\footnotetext{
${ }^{1}$ Faculty of Sport Sciences, Waseda University, 2-579-15 Mikajima, Tokorozawa, Saitama 359-1192, Japan. '2Behavioural Epidemiology Laboratory, Baker Heart and Diabetes Institute, Melbourne, Australia. ${ }^{3}$ Melbourne School of Population and Global Health, The University of Melbourne, Melbourne, Australia. ${ }^{4}$ Faculty of Health and Sport Sciences, University of Tsukuba, Tsukuba, Japan. ${ }^{5}$ Graduate School of Sport Sciences, Waseda University, Tokorozawa, Japan. ${ }^{6}$ Faculty of Liberal Arts and Sciences, Bunka Gakuen University, Tokyo, Japan. ${ }^{7}$ Graduate School of Environmental Studies, Tohoku University, Sendai, Japan. ${ }^{8}$ Department of Community Health Sciences, Cumming School of Medicine, University of Calgary, Calgary, Canada. ${ }^{9}$ Faculty of Kinesiology, University of Calgary, Calgary, Canada. ${ }^{10}$ School of Architecture, Planning and Landscape, University of Calgary, Calgary, Canada. ${ }^{1}$ email: javadkoohsari@aoni.waseda.jp
} 
study conducted in Finland found that dog ownership was associated with higher recreational physical activity in late adulthood ${ }^{21}$. However, this area of research has been limited in several important ways. Most previous studies examining dog ownership and active behaviour used self-reported physical activity measures. A systematic review identified only four studies (out of 29 studies) that used objective measures of physical activity to examine physical activity among dog-owners and non-owners ${ }^{17}$. While self-reported measures of physical activity provide important contextual information, they are limited in some respects. First, self-reported measures of physical activity are subject to recall bias since most people have difficulty in recalling their accurate levels of activities ${ }^{22}$. Second, because physical activity is considered a socially desirable activity, people tend to overreport their physical activity ${ }^{23}$. Finally, self-reported measures of physical activity are unable to accurately capture absolute or total levels of physical activities ${ }^{24}$. To address these issues, there is a need to include objective measures of physical activity, such as accelerometers, in studies comparing time spent in total and intensityspecific physical activity by dog ownership status. Notably, some previous studies used objective physical activity measures in relation to dog ownership using accelerometer devices ${ }^{25-28}$. For example, a study conducted in the USA found a positive association between dog ownership and adolescents' physical activity estimated by accelerometer devices ${ }^{25}$. Another study using accelerometer devices conducted in the UK found that dog walkers were more active than non-owners among a sample of older adults ${ }^{26}$. Nevertheless, these studies are mainly conducted in Western countries, and there is a lack of studies in Asia, where pet ownership is rising ${ }^{29}$. Cultural differences in dog ownership and view of dogs in different geographical locations may impact physical activity decision $^{30,31}$. Additionally, few studies exist examining whether owning a dog may be associated with objectivelymeasured sedentary behaviour ${ }^{25,32,33}$. Sedentary behaviour is an emerging health risk factor independent of physical activity ${ }^{34-37}$ For example, a recent systematic review of prospective studies found that sitting time was associated with an increased risk of cardiovascular disease and diabetes, regardless of physical activity levels ${ }^{36}$. Another systematic review found that total sitting and TV viewing time were associated with higher risk for several major chronic diseases, independent of physical activity ${ }^{37}$. Dog ownership may affect health by reducing people's sedentary behaviour ${ }^{32,38}$. In most of the previous studies, however, self-reported sedentary behaviour was included $^{39}$. For instance, a study conducted in Australia found that dog ownership was not significantly associated with self-reported screen time among children ${ }^{40}$. Further research is needed to explore whether owning a dog may influence adults' objectively-assessed sedentary behaviour or not.

Therefore, our study aimed to investigate the extent to which dog ownership was associated with objectivelyassessed sedentary behaviour and physical activity among adults (40-64 years) living in two Japanese urban areas.

\section{Results}

Complete data from 693 participants were included in this analysis. Our sample included 119 (17.2\%) dogowners. The mean age was 52.2 years, and about $61 \%$ were female, about two-thirds $(64.1 \%)$ had completed tertiary education, about $82 \%$ were employed, and approximately $45 \%$ had an annual gross household income lower than $¥ 5,000,000(\approx$ USD 50,000) (Table 1$)$. The mean accelerometer wearing time was about $15.4(\mathrm{SD}=1.5)$ hours per day, with an average of approximately 7.0 $(\mathrm{SD}=0.9)$ days. Socio-demographic characteristics and accelerometer wearing time/days did not significantly differ between dog-owners and non-owners. Table 2 shows the objectively-assessed sedentary behaviour and physical activity of dog-owners and non-owners. Among dogowners, total sedentary time, duration, and the number of long ( $\geq 30 \mathrm{~min}$ ) sedentary bouts were significantly $(\mathrm{p}<0.05)$ lower compared with non-owners. Sedentary breaks and duration of light-intensity physical activity (min/day) were significantly higher among dog-owners than non-owners. There was no statistically significant difference in moderate, vigorous, and moderate-to-vigorous physical activity between these two groups.

Adjusting for covariates, the means of total sedentary time and the number of long ( $\geq 30 \mathrm{~min})$ sedentary bouts were $26.29 \mathrm{~min} /$ day $(95 \% \mathrm{CI}-47.85,-4.72)$ and 0.41 times/day $(95 \% \mathrm{CI}-0.72,-0.10)$ lower for those who owned a dog compared to those not owning a dog, respectively. Compared with non-owners, dog-owners had significantly higher means of the number of sedentary breaks (95\% CI 0.14, 1.22), and light-intensity physical activity (95\% CI 1.31, 37.51). No significant differences in the duration of long ( $\geq 30 \mathrm{~min})$ sedentary bouts, moderate, vigorous, and moderate-to-vigorous-intensity physical activity were observed between dog-owners and non-owners (Table 3).

\section{Discussion}

We examined the associations between dog ownership with objectively-assessed sedentary behaviour and physical activity among a sample of middle-aged adults in Japan. We found that dog ownership was negatively associated with total sedentary time and number of long sedentary bouts, and positively associated with number of sedentary breaks and light-intensity physical activity. Our findings are consistent with a previous study, which used self-reported measures of sedentary behaviour ${ }^{39}$. Oka and Shibata ${ }^{39}$ found that dog ownership was associated with less daily sedentary behaviour in adults. Some previous studies conducted among older adults also support our findings in part. For instance, owning a dog was associated with a lower likelihood of sitting over $8 \mathrm{~h}$ per day among older women ${ }^{38}$. Another study found that dog-owners had fewer sitting events compared with non-owners. However, they found no significant associations between owning a dog and total sitting time or duration of prolonged sitting events ${ }^{32}$. Our study supports these findings by showing that dog ownership was not only negatively associated with objectively-assessed total sedentary time but with prolonged bouts of sitting. Evidence suggests that both total and prolonged bouts of sitting have adverse health effects ${ }^{41,42}$. Dog ownership may create opportunities for their owners to break their prolonged sedentary behaviour and be engaged in lightintensity physical activities for daily caring for their dogs.

Our findings are in contrast with some previous studies conducted among other age groups. For example, a previous study found that owning a dog was not significantly associated with accelerometer-derived sedentary 


\begin{tabular}{|c|c|c|c|}
\hline \multirow[b]{2}{*}{ Variable } & \multicolumn{3}{|c|}{ Mean (S.D.) or N (\%) } \\
\hline & Total & Dog-owners $(n=119)$ & Non-owners $(n=574)$ \\
\hline Age (years) & $52.2(7.1)$ & $52.5(6.9)$ & $52.1(7.1)$ \\
\hline \multicolumn{4}{|l|}{ Gender } \\
\hline Female & $424(61.2)$ & $72(60.5)$ & $352(61.3)$ \\
\hline Male & $269(38.8)$ & $47(39.5)$ & $222(38.7)$ \\
\hline \multicolumn{4}{|l|}{ Highest education } \\
\hline Tertiary & $444(64.1)$ & $67(56.3)$ & $377(65.7)$ \\
\hline Below tertiary & $249(35.9)$ & $52(43.7)$ & $197(34.3)$ \\
\hline \multicolumn{4}{|l|}{ Working status } \\
\hline Employed & $570(82.3)$ & $103(86.6)$ & $467(81.4)$ \\
\hline Unemployed & $123(17.7)$ & $16(13.4)$ & $107(18.6)$ \\
\hline \multicolumn{4}{|l|}{ Gross annual household income } \\
\hline$<¥ 5,000,000$ & $315(45.5)$ & $55(46.2)$ & $260(45.3)$ \\
\hline$\geq ¥ 5,000,000$ & $378(54.5)$ & $64(53.8)$ & $314(54.7)$ \\
\hline \multicolumn{4}{|l|}{ Municipality } \\
\hline Koto ward & $336(48.5)$ & $39(32.8)$ & $297(51.7)$ \\
\hline Matsuyama city & $357(51.5)$ & $80(67.2)$ & $277(48.3)$ \\
\hline Accelerometer wearing days & $7.0(0.9)$ & $7.1(0.9)$ & $7.0(0.9)$ \\
\hline Accelerometer wearing time (min/day) & $921.2(90.8)$ & $926.0(93.7)$ & $920.2(90.3)$ \\
\hline
\end{tabular}

Table 1. Characteristics of study participants by dog ownership status $(n=693)$. No statistically significant difference between dog-owners and non-dog owners based on independent t-tests and Pearson's chi-square test, except for municipality.

\begin{tabular}{|l|l|l|}
\hline \multirow{2}{*}{} & \multicolumn{2}{|l|}{ Mean (S.D.) } \\
\cline { 2 - 3 } & Dog-owners $(\mathbf{n}=119)$ & Non-owners $(\mathbf{n}=\mathbf{5 7 4})$ \\
\hline Total sedentary time $(\mathrm{min} / \text { day })^{*}$ & $473.1(129.9)$ & $506.3(117.6)$ \\
\hline Duration of long $(\geq 30 \mathrm{~min})$ sedentary bouts $(\mathrm{min})^{*}$ & $155.7(99.0)$ & $175.9(94.0)$ \\
\hline Number of long $(\geq 30 \mathrm{~min})$ sedentary bouts (times/day)* & $2.9(1.6)$ & $3.4(1.6)$ \\
\hline Sedentary breaks (times/sedentary hour)* & $10.1(3.2)$ & $9.2(2.7)$ \\
\hline Light-intensity physical activity $\left(\mathrm{min} /\right.$ day) $^{*}$ & $376.9(115.6)$ & $344.7(109.1)$ \\
\hline Moderate-intensity physical activity $(\mathrm{min} /$ day) & $74.0(40.1)$ & $67.3(37.4)$ \\
\hline Vigorous-intensity physical activity $\left(\mathrm{min} /\right.$ day) $^{*}$ & $1.9(8.8)$ & $1.9(5.6)$ \\
\hline Moderate-to-vigorous-intensity physical activity (min/day) & $75.9(41.7)$ & $69.2(38.7)$ \\
\hline
\end{tabular}

Table 2. Sedentary behaviour and physical activity of dog-owners and non-owners $(n=693)$. Sedentary behaviour, light-intensity physical activity, moderate-intensity physical activity, vigorous-intensity physical activity, and moderate-to-vigorous physical activity were defined with an estimated accelerometer intensity of $\leq 1.5$ METs, 1.6 to 2.9 METs, 3.0-5.9 METs, $\geq 6.0$ METs, and $\geq 3.0$ METs, respectively. ${ }^{*} p$-values based on independent t-tests.

time in a sample of adolescents ${ }^{25}$. Another study found that dog ownership was significantly associated with fewer sitting events in older adults, but not with either the total sitting time or the number or duration of prolonged sedentary events ${ }^{32}$. These findings highlight that dog ownership may have different effects on sedentary behaviours in different age groups. Further research is needed to track how dog ownership may influence sedentary behaviours patterns across the life course.

We found no significant difference in moderate, vigorous, and moderate-to-vigorous-intensity physical activities between dog-owners and non-owners. These findings are in contrast with some previous studies which used self-reported physical activity measures or were conducted in other age groups $s^{20,21,39,43}$. A study conducted in Melbourne, Australia found that owning a dog was associated with higher moderate-to-vigorous-intensity physical activity among children ${ }^{43}$. Another study found that dog-owners were more likely to achieve sufficient levels of physical activity and walking compared with non-owners ${ }^{44}$. Moreover, a study found that dog ownership was associated with higher life course and leisure-time physical activity in late adulthood ${ }^{21}$. There may be several reasons for these inconsistent findings between studies. While much of the physical activity benefits of owning a dog may confer from dog walking, not all dog-owners walk their dogs ${ }^{5}$. The proportion of dog-owners who walked their dogs widely varied between previous studies. For instance, a study conducted in Canada found about $16 \%$ of dog-owners did not walk their $\operatorname{dogs}^{45}$. Another study conducted in the USA reported about $25 \%$ 


\begin{tabular}{|l|c|c|}
\hline & $\begin{array}{l}\text { Unadjusted } \\
\boldsymbol{B}(\mathbf{9 5 \%} \mathbf{C I})\end{array}$ & $\begin{array}{l}\text { Adjusted } \\
\boldsymbol{B}(\mathbf{9 5 \%} \mathbf{C I})\end{array}$ \\
\hline Total sedentary time (min/day) & $-33.14(-56.83,-9.44)^{\star}$ & $-26.29(-47.85,-4.72)^{\star}$ \\
\hline Duration of long $(\geq 30 \mathrm{~min})$ sedentary bouts (min) & $-20.21(-38.98,-1.45)^{\star}$ & $-16.19(-34.68,2.30)$ \\
\hline Number of long ( $\geq 30 \mathrm{~min})$ sedentary bout (times/day) & $-0.48(-0.80,-0.17)^{\star}$ & $-0.41(-0.72,-0.10)^{\star}$ \\
\hline Number of sedentary breaks (times/sedentary hour) & $0.88(0.33,1.43)^{\star}$ & $0.68(0.14,1.22)^{\star}$ \\
\hline Light-intensity physical activity (min/day) & $32.21(10.42,54.01)^{\star}$ & $19.41(1.31,37.51)^{\star}$ \\
\hline Moderate-intensity physical activity (min/day) & $6.67(-0.82,14.16)$ & $6.54(-0.92,14.01)$ \\
\hline Vigorous-intensity physical activity (min/day) & $0.02(-1.21,1.26)$ & $0.33(-0.90,1.57)$ \\
\hline Moderate-to-vigorous-intensity physical activity (min/day) & $6.69(-1.06,14.44)$ & $6.88(-0.85,14.61)$ \\
\hline
\end{tabular}

Table 3. Associations of dog ownership with objectively-assessed sedentary behaviour and physical activity $(n=693)$. All models adjusted for age, gender, highest education, working status, gross annual household income, municipality, and accelerometer wearing time. Reference group: non-dog owners. $B$ regression unstandardised coefficient, $C I$ confidence interval. ${ }^{*} \mathrm{p}<0.05$.

of their participants as dog-owners non walkers ${ }^{46}$. A recent study of Japanese adults showed that about $30 \%$ of dog-owners did not walk their dogs in a usual week ${ }^{47}$. Additionally, there might be socio-cultural or urban design factors that act to discourage dog-owners from walking their dogs in the context of our Japanese adults. Since Japan has one of the most prolonged working hours in the world ${ }^{48}$, dog-owners may have limited time to devote to walking and playing outside with their dogs. Unique urban design attributes of Japanese urban environments such as narrow sidewalks, small housing, and lack of dog-friendly parks may also deter dog-owners from undertaking dog-related medium-to-high intensity physical activities. Furthermore, and relevant to the compact built environment, small dog breeds are popular among dog-owners in Japan ${ }^{49}$. According to the Japan Pet Food Association's survey, more than half of Japanese dog-owners have a small-sized $\operatorname{dog}^{50}$. Such breeds may require less physical activities compared with their larger breeds ${ }^{51}$. Further studies are needed to identify correlates of higher intensity physical activities among dog-owners in this context.

Our study has some limitations. As a cross-sectional study, it cannot provide strong causal evidence. Dog characteristics (such as age, sizes, breeds) and owner-dog attachment or bond were not considered in this study. Objective measures of sedentary behaviour and physical activity do not provide contextual information on the specific types of these activities. Therefore, the extent to which dog-owners physical activity included dog walking was not captured. A key strength of this study is the use of objectively-assessed sedentary behaviour and physical activity. The examination of physical activity at different intensities is another strength of this study.

\section{Conclusions}

This study adds to the existing literature on dog ownership and active and sedentary behaviours by using objectively-assessed behaviours. Our findings can contribute to a better understanding of how dog ownership may impact adults' active and sedentary behaviours. A novel result of this study is that owning a dog was associated with several types of adults' sedentary behaviours but not medium-to-high-intensity physical activities. These findings provide several new insights for dog-based behavioural health interventions: first, they highlight the importance of dog ownership for reducing adults' sedentary behaviour, as an emerging health risk. Dog ownership status appears to be associated with frequency of bouts and time spent sedentary. Notably, a previous review reported the importance of various strategies to encourage dog walking in order to improve health ${ }^{18}$. However, our findings suggest that lower levels of sedentary behaviour, in addition to increased physical activity accumulated via dog walking, might be another pathway by which dog owners accumulate health benefits. Second, our findings indicate that other factors, as well as dog ownership, are necessary to affect dog owners' mediumto-high-intensity physical activities. Finally, dog ownership status is an important correlate of physical activity and sedentary behaviour in Japanese middle-aged adults and therefore should be considered in the design and planning of health interventions and in studies investigating the determinants of physical activity and sedentary behaviour in this target population.

\section{Methods}

Study and sample design. Our analysis included cross-sectional data from an epidemiological study undertaken to examine social and urban design correlates of sedentary behaviour and physical activity among Japanese adults aged 40-64 years. Detailed methods of study design and recruitment have been documented elsewhere ${ }^{52}$. Briefly, data were obtained between July to December 2013 and April 2014 to February 2015 from residents living in two Japanese urban localities, Koto Ward and Matsuyama City. To recruit participants, an invitation letter was sent to 6,000 adult residents (aged 40-64 years), randomly selected from the government registry of residential addresses. About two weeks after the initial mailing, a reminder letter was sent to nonrespondents. A self-administered questionnaire and an accelerometer (with a log diary) were posted to the 866 individuals (response rate $=14.4 \%$ ), who agreed to participate in this study. Of these, 779 completed the questionnaire and returned the accelerometers. A book voucher (¥1000 equivalent to about USD\$10) was given to these participants. The study was approved by the Research Ethics Committee, Waseda University, Japan (2012- 
269) and the methods were carried out in accordance with these guidelines. Informed consent was obtained from each participant.

Measures. Sedentary behaviour and physical activity. Sedentary behaviour and physical activity were objectively-assessed using a validated tri-axial accelerometer (Active style Pro model HJA-350IT; Omron Healthcare, Kyoto, Japan $)^{53,54}$. Participants were asked to wear the accelerometer on their waist for at least seven days while they are awake, except during water-based activities (e.g., bathing, showering, swimming). Participants who wore the accelerometer for at least four days (including one non-working day), with at least $10 \mathrm{~h} / \mathrm{day}$ of wear time per day, were eligible for this study ${ }^{55}$. The data were collected in 1 -min epochs and expressed as metabolic equivalents (METs). Sedentary behaviour, light-intensity physical activity, moderate-intensity physical activity, vigorous-intensity physical activity, and moderate-to-vigorous physical activity were defined with an estimated accelerometer intensity of $\leq 1.5$ METs, 1.6 to 2.9 METs, 3.0-5.9 METs, $\geq 6.0$ METs, and $\geq 3.0$ METs, respectively ${ }^{56,57}$. Four sedentary behaviour outcomes, including total sedentary time, duration and number of long ( $\geq 30 \mathrm{~min}$ ) sedentary bouts and breaks per sedentary hour, were calculated. The total sedentary time per day was obtained by summing the time spent engaged in any sedentary behaviour ${ }^{57}$. Sedentary bouts were defined as periods of uninterrupted sedentary time and a sedentary bout referred to at least 30 consecutive minutes of sedentary time $\mathrm{e}^{57,58}$. A sedentary break was defined as a non-sedentary bout between two sedentary bouts ${ }^{57}$. Physical activity outcomes were averages of light, moderate, vigorous, and moderate-to-vigorous-intensity physical activity per day.

Dog ownership. Participants were asked whether they currently own a pet in their household or not. Those who owned a pet reported the type of pet, including dog, cat, and others (e.g., birds, fish, and reptile). We compared those with (owners) and without (non-owners) a dog at home only.

Covariates. Participants reported the following socio-demographic characteristics: age, gender (female versus male), highest educational attainment (tertiary versus below tertiary), working status (employed versus unemployed), and annual gross household income $(<¥ 5,000,000$ versus $\geq ¥ 5,000,000)$. The municipality (Koto Ward versus Matsuyama City) and daily accelerometer wearing time were also included as covariates.

Statistical analysis. Descriptive statistics including frequencies and measures of central tendency and variation (i.e., means, standard deviations) were estimated for socio-demographic, dog ownership, sedentary behaviour, and physical activity variables. Independent t-tests and Pearson's chi-square test were used to compare these variables between dog-owners and non-owners. Multivariable linear regression models were used to estimate associations of dog ownership with objectively-assessed sedentary behaviour and physical activity. All models adjusted for the covariates. Analyses were conducted using Stata 15.0 (Stata Corp, College Station, Texas), and the level of significance was set at $\mathrm{p}<0.05$.

Received: 6 May 2020; Accepted: 25 September 2020

Published online: 15 October 2020

\section{References}

1. Matchock, R. L. Pet ownership and physical health. Curr. Opin. Psychiatry 28, 386-392 (2015).

2. Levine, G. N. et al. Pet ownership and cardiovascular risk: A scientific statement from the American Heart Association. Circulation 127, 2353-2363 (2013).

3. Mubanga, M. et al. Dog ownership and the risk of cardiovascular disease and death-A nationwide cohort study. Sci. Rep. 7, 15821 (2017).

4. Krittanawong, C. et al. Pet ownership and cardiovascular health in the US general population. Am. J. Cardiol. https://doi. org/10.1016/j.amjcard.2020.01.030 (2020).

5. Christian, H. E., McCormack, G. R., Evenson, K. R. \& Maitland, C. Dog walking. In Walking: Connecting Sustainable Transport with Health (Transport and Sustainability) (eds Corinne Mulley, K. G. \& Ding, D.) (Emerald Publishing Limited, Bingley, 2017).

6. Barcelos, A. M., Kargas, N., Maltby, J., Hall, S. \& Mills, D. S. A framework for understanding how activities associated with dog ownership relate to human well-being. Sci. Rep. 10, 1-12 (2020).

7. Warburton, D. E. \& Bredin, S. S. Health benefits of physical activity: A systematic review of current systematic reviews. Curr. Opin. Cardiol. 32, 541-556 (2017).

8. Guthold, R., Stevens, G. A., Riley, L. M. \& Bull, F. C. Worldwide trends in insufficient physical activity from 2001 to $2016:$ A pooled analysis of 358 population-based surveys with 1.9 million participants. Lancet. Glob. Health 6, e1077-1086 (2018).

9. Blackwell, D. L. \& Clarke, T. C. State variation in meeting the 2008 federal guidelines for both aerobic and muscle-strengthening activities through leisure-time physical activity among adults aged 18-64: United States, 2010-2015. Natl. Health. Stat. Rep. 112, $1-22(2018)$.

10. Inoue, S. et al. Time trends for step-determined physical activity among Japanese adults. Med. Sci. Sports. Exerc. 43, 1913-1919 (2011).

11. World Health Organization. Prevalence of insufficient physical activity among adults: Data by country. Glob. Heal. Obs. data Repos. (2016).

12. Sallis, J. F. \& Owen, N. Ecological models of health behaviour. In Health Behavior Theory Research and Practice (eds Glanz, K. et al.) 43-64 (Jossey-Bass, San Francisco, 2015)

13. Growth from Knowledge. Pet ownership global GfK survey. (2016).

14. Canadian Veterinary Medical Association. Canada's Pet Wellness Report (Canadian Veterinary Medical Association, Ottawa, 2011).

15. American Pet Products Association. APPA National Pet Owners Survey 2017-2018 (APPA, Greenwich, 2018).

16. Australia Animal Medicines. Pet ownership in Australia. Summary 2016. (Animal Medicines Australia, Barton, 2016).

17. Christian, H. E. et al. Dog ownership and physical activity: A review of the evidence. J. Phys. Act. Health 10, 750-759 (2013).

18. Christian, H. et al. Encouraging dog walking for health promotion and disease prevention. Am. J. Lifestyle Med. 12, 233-243 (2018). 
19. Johnson, R. A., Beck, A. M. \& McCune, S. K. The Health Benefits of Dog Walking for Pets and People: Evidence and Case Studies (Purdue University Press, West Lafayette, 2011).

20. Westgarth, C. et al. Dog owners are more likely to meet physical activity guidelines than people without a dog: An investigation of the association between dog ownership and physical activity levels in a UK community. Sci. Rep. 9, 1-10 (2019).

21. Wasenius, N. S. et al. Dog ownership from a life course perspective and leisure-time physical activity in late adulthood: The Helsinki Birth Cohort study. Am. J. Health Behav. 42, 11-18 (2018).

22. Shephard, R. J. Limits to the measurement of habitual physical activity by questionnaires. Br. J. Sports Med. 37, 197-206 (2003).

23. Sallis, J. F. \& Saelens, B. E. Assessment of physical activity by self-report: Status, limitations, and future directions. Res. Q. Exerc. Sport. 71, 1-14 (2000).

24. Prince, S. A. et al. A comparison of direct versus self-report measures for assessing physical activity in adults: A systematic review. Int. J. Behav. Nutr. Phys. Act. 5, 56 (2008).

25. Sirard, J. R., Patnode, C. D., Hearst, M. O. \& Laska, M. N. Dog ownership and adolescent physical activity. Am. J. Prev. Med. 40, 334-337 (2011).

26. Wu, Y.-T., Luben, R. \& Jones, A. Dog ownership supports the maintenance of physical activity during poor weather in older English adults: Cross-sectional results from the EPIC Norfolk cohort. J. Epidemiol. Community Health 71, 905-911 (2017).

27. Engelberg, J. K. et al. Dog walking among adolescents: Correlates and contribution to physical activity. Prev. Med. 82, 65-72 (2016).

28. Brown, B. B. \& Jensen, W. A. Dog ownership and walking: Perceived and audited walkability and activity correlates. Int. J. Environ. Res. Public Health. 17, 1385 (2020).

29. Spire Research and Consulting. The Pet Economy Boom in Asia (Spire Research and Consulting, Gurugram, 2018).

30. Dotson, M. J. \& Hyatt, E. M. Understanding dog-human companionship. J. Bus. Res. 61, 457-466 (2008).

31. Blouin, D. D. Are dogs children, companions, or just animals? understanding variations in people's orientations toward animals. Anthrozoös 26, 279-294 (2013).

32. Dall, P. M. et al. The influence of dog ownership on objective measures of free-living physical activity and sedentary behaviour in community-dwelling older adults: A longitudinal case-controlled study. BMC Public Health 17, 1-9 (2017).

33. White, M. N. et al. Caregiving, transport-related, and demographic correlates of sedentary behavior in older adults: The senior neighborhood quality of life study. J. Aging Health 28, 812-833 (2016).

34. Xu, C. et al. Sedentary behavior, physical activity, and all-cause mortality: Dose-response and intensity weighted time-use metaanalysis. J. Am. Med. Dir. Assoc. 20, 1206-1212 (2019).

35. Wang, X., Li, Y. \& Fan, H. The associations between screen time-based sedentary behavior and depression: A systematic review and meta-analysis. BMC Public Health 19, 1524 (2019).

36. Bailey, D. P., Hewson, D. J., Champion, R. B. \& Sayegh, S. M. Sitting time and risk of cardiovascular disease and diabetes: A systematic review and meta-analysis. Am. J. Prev. Med. 57, 408-416 (2019).

37. Patterson, R. et al. Sedentary behaviour and risk of all-cause, cardiovascular and cancer mortality, and incident type 2 diabetes: A systematic review and dose response meta-analysis. Eur. J. Epidemiol. 33, 811-829 (2018).

38. Garcia, D. O. et al. Relationships between dog ownership and physical activity in postmenopausal women. Prev. Med. 70, 33-38 (2015).

39. Oka, K. \& Shibata, A. Dog ownership and health-related physical activity among Japanese adults. J. Phys. Act. Health 6, 412-418 (2009).

40. Christian, H., Trapp, G., Lauritsen, C., Wright, K. \& Giles-Corti, B. Understanding the relationship between dog ownership and children's physical activity and sedentary behaviour. Pediatr. Obes. 8, 392-403 (2013).

41. Duvivier, B. M. et al. Breaking sitting with light activities vs structured exercise: A randomised crossover study demonstrating benefits for glycaemic control and insulin sensitivity in type 2 diabetes. Diabetologia 60, 490-498 (2017).

42. Credeur, D. P. et al. Impact of prolonged sitting on peripheral and central vascular health. Am. J. Cardiol. 123, 260-266 (2019).

43. Salmon, J., Timperio, A., Chu, B. \& Veitch, J. Dog ownership, dog walking, and children's and parents' physical activity. Res. Q. Exerc. Sport. 81, 264-271 (2010).

44. Cutt, H., Giles-Corti, B., Knuiman, M., Timperio, A. \& Bull, F. Understanding dog owners' increased levels of physical activity: Results from RESIDE. Am. J. Public Health 98, 66-69 (2008).

45. McCormack, G. R., Rock, M., Sandalack, B. \& Uribe, F. A. Access to off-leash parks, street pattern and dog walking among adults. Public Health 125, 540-546 (2011).

46. Richards, E. A. Does dog walking predict physical activity participation: Results from a national survey. Am. J. Health Promot. 30, 323-330 (2016).

47. Koohsari, M. J. et al. Dog-walking in dense compact areas: The role of neighbourhood built environment. Health Place 61, 102242 (2019).

48. Ono, H. Why do the Japanese work long hours. Sociological perspectives on long working hours in Japan. Japan Labor Issues 2 , $35-49(2018)$.

49. Iris Ohyama Inc. Dog Census 2018 (Iris Ohyama Inc, Sendai, 2018).

50. Japan Pet Food Association. Research for the Number of Household Dog and Cats in Japan (Japan Pet Food Association, Tokyo, 2016).

51. Degeling, C., Burton, L. \& McCormack, G. R. An investigation of the association between socio-demographic factors, dog-exercise requirements, and the amount of walking dogs receive. Can. J. Vet. Res. 76, 235-240 (2012).

52. Ishii, K. et al. Validity and reliability of Japanese-language self-reported measures for assessing adults domain-specific sedentary time. J. Epidemiol. https://doi.org/10.2188/jea.JE20170002 (2017).

53. Ohkawara, K. et al. Real-time estimation of daily physical activity intensity by a triaxial accelerometer and a gravity-removal classification algorithm. Br. J. Nutr. 105, 1681-1691 (2011).

54. Oshima, Y. et al. Classifying household and locomotive activities using a triaxial accelerometer. Gait Posture 31, 370-374 (2010).

55. Healy, G. N., Matthews, C. E., Dunstan, D. W., Winkler, E. A. \& Owen, N. Sedentary time and cardio-metabolic biomarkers in US adults: NHANES 2003-06. Eur. Heart. J. 32, 590-597 (2011).

56. Ainsworth, B. E. et al. 2011 compendium of physical activities: A second update of codes and MET values. Med. Sci. Sports. Exerc. 43, 1575-1581 (2011).

57. Tremblay, M. S. et al. Sedentary behavior research network (SBRN)-terminology consensus project process and outcome. Int. J. Behav. Nutr. Phys. Act. 14, 75 (2017).

58. Thorp, A. A. et al. Prolonged sedentary time and physical activity in workplace and non-work contexts: A cross-sectional study of office, customer service and call centre employees. Int. J. Behav. Nutr. Phys. Act. 9, 128 (2012).

\section{Acknowledgements}

Oka is supported by a Grant-in-Aid for Scientific Research (No. 20H04113) from the Japan Society for the Promotion of Science. 


\section{Author contributions}

M.J.K., A.S. and K.O. conceived the idea, analysed the data, and drafted the paper. T.N. assisted with the statistical analysis. K.I., S.K., A.Y., T.H., T.N. and G.L.M. contributed to the writing and assisted with the interpretation. All authors reviewed and approved the final manuscript.

\section{Competing interests}

The authors declare no competing interests.

\section{Additional information}

Correspondence and requests for materials should be addressed to M.J.K.

Reprints and permissions information is available at www.nature.com/reprints.

Publisher's note Springer Nature remains neutral with regard to jurisdictional claims in published maps and institutional affiliations.

(c) (i) Open Access This article is licensed under a Creative Commons Attribution 4.0 International License, which permits use, sharing, adaptation, distribution and reproduction in any medium or format, as long as you give appropriate credit to the original author(s) and the source, provide a link to the Creative Commons licence, and indicate if changes were made. The images or other third party material in this article are included in the article's Creative Commons licence, unless indicated otherwise in a credit line to the material. If material is not included in the article's Creative Commons licence and your intended use is not permitted by statutory regulation or exceeds the permitted use, you will need to obtain permission directly from the copyright holder. To view a copy of this licence, visit http://creativecommons.org/licenses/by/4.0/.

(C) The Author(s) 2020 